\title{
DENSITY OF CLASSICAL POINTS IN EIGENVARIETIES
}

\author{
DAVID LOEFFLER
}

\begin{abstract}
In this short note, we study the geometry of the eigenvariety parametrizing $p$-adic automorphic forms for $\mathrm{GL}_{1}$ over a number field, as constructed by Buzzard. We show that if $K$ is not totally real and contains no CM subfield, points in this space arising from classical automorphic forms (i.e. algebraic Grössencharacters of $K$ ) are not Zariskidense in the eigenvariety (as a rigid space); but the eigenvariety posesses a natural formal scheme model, and the set of classical points is Zariski-dense in the formal scheme.

We also sketch the theory for $\mathrm{GL}_{2}$ over an imaginary quadratic field, following Calegari and Mazur, emphasizing the strong formal similarity with the case of $\mathrm{GL}_{1}$ over a general number field.
\end{abstract}

\section{Introduction}

In the influential paper [4], Coleman and Mazur constructed a $p$-adic rigid analytic space (the eigencurve) parametrizing the Hecke eigenvalues of overconvergent $p$-adic modular eigenforms. More recently, analogues of the eigencurve, known as eigenvarieties, have been constructed which interpolate Hecke eigenvalues of automorphic representations for a wide range of reductive groups (see e.g. [1, 6, 9]).

For the Coleman-Mazur eigencurve, it is known that the set of points corresponding to classical modular eigenforms (classical points) is a Zariski-dense subset. The two ingredients in the proof of this result are Coleman's classicality criterion for overconvergent eigenforms [3], and the fact that the map from the eigencurve to the underlying $p$-adic weight space is locally finite and flat. For more general eigenvarieties, one has analogues of the first fact (e.g. [9, Theorem 3.9.6]), but it is not always the case that the map to weight space is flat, so one cannot deduce that classical points are dense in the eigenvariety.

In this note, we investigate in detail a special case where these phenomena can be studied more explicitly: the case of $\mathrm{GL}_{1}$ over a number field $K$. In this case an explicit description of the eigenvariety has been given by Buzzard in [1]. It follows easily from this description that if $K$ is neither $\mathbb{Q}$ nor an imaginary quadratic field, then the map from the eigenvariety to weight space is not flat. We show that if moreover $K$ is not totally real and has no CM subfield, then the set of classical points is not Zariskidense in the eigenvariety (considered as a rigid space). However, we show that the eigenvariety has a natural formal scheme structure, and the set of classical points is always Zariski-dense in the eigenvariety when considered as a formal scheme.

This investigation was motivated by the results of [2] for $\mathrm{GL}_{2}$ over an imaginary quadratic field, where somewhat similar phenomena occur. In this setting one works with the "ordinary eigenvariety" constructed by Hida [8]. (This ordinary eigenvariety

Received by the editors September 6, 2010. Revision received April 4, 2011.

Supported by EPSRC postdoctoral fellowship EP/F04304X/2. 
should be a component of the eigenvariety given by the machinery of [6], although the details of this construction do not appear to have been written down.) In Section 5, we recall the results of [2] which show that classical points are not dense in the ordinary eigenvariety when considered as a rigid space. However, this ordinary eigenvariety also carries a natural formal scheme structure, and we show that the results of op.cit. are not sufficient to rule out the possibility that some component of this space contains infinitely many classical points (and hence that classical points are dense in that component when considered as a formal scheme).

\section{Zariski density in formal and rigid spaces}

Let $A$ be a finite algebra over the formal power series ring $\mathbb{Z}_{p} \llbracket T_{1}, \ldots, T_{n} \rrbracket$ for some $n \geq 0$, which is flat over $\mathbb{Z}_{p}$ (i.e. $p$ is not a zero-divisor in $A$ ). Then we have a choice of geometric objects attached to $A$ : the affine scheme $\operatorname{Spec}(A)$, and its generic fibre $\operatorname{Spec}\left(A\left[\frac{1}{p}\right]\right)$; the affine formal scheme $\operatorname{Spf}(A)$; and the rigid-analytic space $(\operatorname{Spf}(A))^{\text {rig }}$ obtained by applying Berthelot's generic fibre construction [5, Section 7]. We abbreviate the latter by $\operatorname{Rig}(A)$.

Proposition 2.1. The following three sets are in canonical bijection with each other:

- Points of $\operatorname{Spec}\left(A\left[\frac{1}{p}\right]\right)$, i.e., maximal ideals of $A\left[\frac{1}{p}\right]$;

- Points of $\operatorname{Rig}(A)$;

- Equivalence classes of morphisms of formal schemes $\operatorname{Spf}(\mathcal{O}) \rightarrow \operatorname{Spf}(A)$, with $\mathcal{O}$ the ring of integers of a finite extension of $\mathbb{Q}_{p}$. Here two morphisms $\operatorname{Spf}(\mathcal{O}) \rightarrow \operatorname{Spf}(A)$ and $\operatorname{Spf}\left(\mathcal{O}^{\prime}\right) \rightarrow \operatorname{Spf}(A)$ are equivalent if there exists a third such ring $\mathcal{O}^{\prime \prime}$ and a commutative diagram

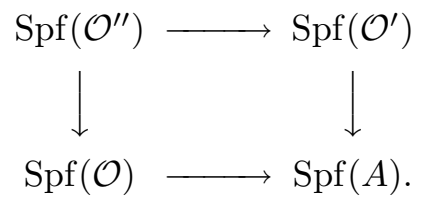

Proof. See [5, Sections 7.1.9-7.1.10].

We do not, however, obtain bijections between closed subvarieties of these geometric objects; closed subschemes of $\operatorname{Spec}\left(A\left[\frac{1}{p}\right]\right)$ biject with closed formal subschemes of $\operatorname{Spf} A$ flat over $\mathbb{Z}_{p}$, but these correspond to a subset of the closed subvarieties of $\operatorname{Rig}(A)$. Most of the content of the present note relates in some way or another to the following key example. If $A=\mathbb{Z}_{p} \llbracket T \rrbracket$, then $\operatorname{Rig}(A)$ is the rigid-analytic open unit disc, and the set of points $T$ such that $(1+T)^{p^{n}}=1$ for some $n \in \mathbb{N}$ is a closed subvariety of $\operatorname{Rig}(A)$ (cut out by the $p$-adic $\operatorname{logarithm} \log (1+T)$ ), which is clearly not the analytification of any closed subvariety of $\operatorname{Spf}(A)$.

If $P(A)$ is the common set of points from the preceding proposition, we refer to the topology on $P(A)$ whose closed subsets are given by ideals of $A\left[\frac{1}{p}\right]$ (or, equivalently, closed subvarieties of $\operatorname{Spf}(A)$ flat over $\left.\mathbb{Z}_{p}\right)$ as the formal Zariski topology, and the topology whose closed subsets are given by rigid-analytic subvarieties of $\operatorname{Rig}(A)$ as the rigid Zariski topology. As the preceding example shows the rigid Zariski topology may be strictly finer than the formal Zariski topology. 


\section{Character spaces}

Let $G$ be a compact abelian $p$-adic analytic group. Since any such group has a subgroup of finite index isomorphic to $\mathbb{Z}_{p}^{d}$ for some $d$, it is topologically finitely generated. Hence, it is isomorphic to a direct sum of finitely many procyclic subgroups [10, Theorem 4.3.5]. Since each factor must also be $p$-adic analytic, each of these procyclic subgroups must be either finite or isomorphic to $\mathbb{Z}_{p}$, and we see that $G$ is isomorphic to $\mathbb{Z}_{p}^{d} \times H$ for some finite abelian group $H$.

Theorem 3.1. For any group $G$ as above, the functor mapping an Artinian local $\mathbb{Z}_{p}$-algebra $A$ to the set of continuous group homomorphisms $G \rightarrow A^{\times}$is prorepresentable, and is represented by the formal scheme $\widehat{G}=\operatorname{Spf} \mathbb{Z}_{p} \llbracket G \rrbracket$, where $\mathbb{Z}_{p} \llbracket G \rrbracket$ is the Iwasawa algebra of $G$, equipped with the canonical character $G \rightarrow \mathbb{Z}_{p} \llbracket G \rrbracket^{\times}$. Moreover, the generic fibre $\widehat{G}^{\text {rig }}$ of $\widehat{G}$ is the rigid space constructed in [1, Lemma 2] which represents the corresponding functor on the category of affinoid $\mathbb{Q}_{p}$-algebras.

Proof. Essentially by definition, any continuous homomorphism $G \rightarrow A^{\times}$extends uniquely to a ring homomorphism $\mathbb{Z}_{p} \llbracket G \rrbracket \rightarrow A$, and conversely any ring homomorphism $\mathbb{Z}_{p} \llbracket G \rrbracket \rightarrow A$ gives a group homomorphism $G \rightarrow A^{\times}$by composition with the canonical character (which is continuous, since $A$ is Artinian). Furthermore, $\mathbb{Z}_{p} \llbracket G \rrbracket$ can clearly be written as an inverse limit of the quotients $\left(\mathbb{Z} / p^{n} \mathbb{Z}\right) \llbracket G / U \rrbracket$ for $U$ open in $G$, which are Artinian $\mathbb{Z}_{p}$-algebras. Moreover, if $G_{1}$ and $G_{2}$ are two such groups, we have

$$
\mathbb{Z}_{p} \llbracket G_{1} \times G_{2} \rrbracket=\mathbb{Z}_{p} \llbracket G_{1} \rrbracket \hat{\otimes}_{\mathbb{Z}_{p}} \mathbb{Z}_{p} \llbracket G_{2} \rrbracket ;
$$

the generic fibre construction commutes with fibre products, so it suffices to check that the generic fibre of $\operatorname{Spf} \mathbb{Z}_{p} \llbracket G \rrbracket$ agrees with Buzzard's construction when $G$ is either $\mathbb{Z}_{p}$ or a finite cyclic group; both of these cases are easy.

Now let $K$ be a number field. We define

$$
\mathcal{O}_{K, p}^{\times}:=\left(\mathcal{O}_{K} \otimes \mathbb{Z}_{p}\right)^{\times}=\prod_{v \mid p} \mathcal{O}_{K, v}^{\times} .
$$

It is clear that $\mathcal{O}_{K, p}^{\times}$is an abelian $p$-adic analytic group of dimension $d=[K: \mathbb{Q}]$; we let $\mathcal{W}=\widehat{\mathcal{O}_{K, p}^{\times}}$. A point of $\mathcal{W}$ is thus equivalent to a continuous homomorphism $\mathcal{O}_{K, p}^{\times} \rightarrow E^{\times}$, for $E$ some finite extension of $\mathbb{Q}_{p}$; we refer to these as $p$-adic weights for $K$.

Let $K_{\infty}^{\circ}$ be the identity component of $(K \otimes \mathbb{R})^{\times}$, and $U$ any open compact subgroup of $\left(\mathbb{A}_{K}^{p, \infty}\right)^{\times}$. We define

$$
H(U)=\mathbb{A}_{K}^{\times} / \overline{K^{\times} \cdot U \cdot K_{\infty}^{\circ}}
$$

Definition 3.1. [1, Section 2] The eigenvariety for $\mathrm{GL}_{1} / K$ of tame level $U$ is the formal $\mathbb{Z}_{p}$-scheme $\mathcal{E}(U)=\widehat{H(U)}$.

The inclusion $\mathcal{O}_{K, p}^{\times} \hookrightarrow \mathbb{A}_{K}^{\times}$gives a continous map $\mathcal{O}_{K, p}^{\times} \rightarrow H(U)$ whose kernel is the closure in $\mathcal{O}_{K, p}^{\times}$of the abelian group $\Gamma(U)=K^{\times} \cap\left(U \cdot \mathcal{O}_{K, p}^{\times} \cdot K_{\infty}^{\circ}\right)$. The cokernel of this map is finite (it is the ray class group modulo $U K_{\infty}^{\circ}$ ) and hence $H(U)$ is also a compact abelian $p$-adic analytic group, of dimension equal to $1+r_{2}(K)+\delta(K)$ where 
$r_{2}(K)$ is the number of complex places of $K$ and $\delta(K)$ is the defect in Leopoldt's conjecture for $K$ at $p$.

If we write $Q(U)=\mathcal{O}_{K, p}^{\times} / \overline{\Gamma(U)}$, then we can identify $Q(U)$ with a finite-index subgroup of $H(U)$; hence, we have maps $\mathbb{Z}_{p} \llbracket \mathcal{O}_{K, p}^{\times} \rrbracket \rightarrow \mathbb{Z}_{p} \llbracket Q(U) \rrbracket \hookrightarrow \mathbb{Z}_{p} \llbracket H(U) \rrbracket$, where the second map is finite and flat (and becomes étale after inverting $p$ ). Thus, the morphism $\mathcal{E}(U) \rightarrow \mathcal{W}$ factors as a finite flat surjective map followed by the inclusion of the closed subscheme $\mathcal{W}(U)=\widehat{Q(U)}$ of $\mathcal{W}$. In particular, we have the following result:

Proposition 3.1. Every component of $\mathcal{E}(U)$ has dimension equal to $1+r_{2}+\delta$, and maps surjectively to a component of $\mathcal{W}(U)$.

(Note that $\mathcal{E}(U)$ is not flat over $\mathcal{W}$ unless $\mathcal{O}_{K}^{\times}$is finite, i.e., $K$ is either $\mathbb{Q}$ or an imaginary quadratic field.)

\section{Algebraic points}

Let us fix embeddings of $\overline{\mathbb{Q}}$ into $\overline{\mathbb{Q}}_{p}$ and into $\mathbb{C}$. Let $\kappa$ be a $p$-adic weight for $K$. We say that $\kappa$ is algebraic if we can write

$$
\kappa(x)=\prod_{i} \sigma_{i}(x)^{n_{i}}
$$

where $\sigma_{1}, \ldots, \sigma_{d}$ are the ring homomorphisms $\mathcal{O}_{K, p} \rightarrow \overline{\mathbb{Q}}_{p}$ arising from the $d$ embeddings $K \hookrightarrow \overline{\mathbb{Q}}$, and $n_{i} \in \mathbb{Z}$. If $\kappa$ is algebraic in the above sense when restricted to some open neighbourhood of the identity, we say $\kappa$ is locally algebraic; this is equivalent to the existence of a factorization $\kappa=\varepsilon \kappa^{\prime}$ where $\kappa^{\prime}$ is algebraic and $\varepsilon$ has finite order.

The norm map $N_{K / \mathbb{Q}}$ extends continuously to a homomorphism $\mathcal{O}_{K, p}^{\times} \rightarrow \mathbb{Z}_{p}^{\times}$. We say $\kappa$ is parallel if it factors through this map; we say $\kappa$ is locally parallel if this holds on some open neighbourhood of the identity. Note that an algebraic weight is parallel if and only if the $n_{i}$ are all equal.

Our choice of embeddings gives a bijection between algebraic Grössencharacters of $K$ (of level $U$ ) and points of $\mathcal{E}(U)$ whose projection to $\mathcal{W}$ is locally algebraic. This maps a Grössencharacter of infinity-type $x \mapsto \prod_{i} \sigma_{i}(x)^{n_{i}}$ to a locally algebraic character with the same algebraic part.

For the rest of this section, let us make the following assumption:

Assumption. The field $K$ contains no $C M$ subfield.

Theorem 4.1. [11] If the above assumption holds, then the infinity-type of every algebraic Grössencharacter of $K$ is parallel.

If $\kappa$ is a locally algebraic weight, we define $c(\kappa)$ to be the smallest integer $r \geq 0$ such that $\kappa$ is algebraic when restricted to $1+p^{r} \mathcal{O}_{K, p}$.

Proposition 4.1. For any $N<\infty$, there is a one-dimensional closed formal subscheme of $\mathcal{W}$ that contains every locally algebraic weight $\kappa \in \mathcal{W}(U)$ with $c(\kappa) \leq N$.

Proof. If $\kappa \in \mathcal{W}(U)$ is locally algebraic, then by Weil's theorem it must be of the form $x \mapsto \varepsilon(x) N_{K / \mathbb{Q}}(x)^{k}$ for some $k \in \mathbb{Z}$ and finite-order $\varepsilon$. We may assume without loss of generality that $N_{K / \mathbb{Q}}(\Gamma(U))=\{1\}$, so $\varepsilon$ and $N_{K / \mathbb{Q}}(x)^{k}$ both lie in $\mathcal{W}(U)$. 
Since the subgroup $\overline{\Gamma(U)} \cdot\left(1+p^{N} \mathcal{O}_{K, p}\right)$ has finite index in $\mathcal{O}_{K, p}^{\times}$, there are only finitely many candidates for $\varepsilon$. Hence the locally algebraic weights with $c(\kappa) \leq N$ are contained in the union of finitely many translates of the one-dimensional subscheme $\mathcal{W}_{0} \subseteq \mathcal{W}$ parametrizing parallel weights (which is simply the space of characters of $\left.N_{K / \mathbb{Q}}\left(\mathcal{O}_{K, p}^{\times}\right) \subseteq \mathbb{Z}_{p}^{\times}\right)$.

We assume henceforth that $K$ is not totally real, so $\mathcal{W}(U)$ has dimension $1+r_{2}+\delta>$ 1. It follows that the locally algebraic weights with $c(\kappa) \leq N$ are not dense in the formal Zariski topology of $\mathcal{W}(U)$. In particular, for a fixed coefficient field $E$ which is discretely valued, the set of $E$-valued finite-order characters is finite (since $E$ contains finitely many $p$-power roots of unity) and thus the locally algebraic $E$-valued weights are not formally Zariski-dense in $\mathcal{W}(U)$.

Proposition 4.2. The closure of the locally algebraic weights in the rigid Zariski topology of $\mathcal{W}(U)$ is a closed rigid subvariety of $W(U)$ of dimension 1. However, this set is dense in the formal Zariski topology of $\mathcal{W}(U)$.

Proof. Let $u_{1}, \ldots, u_{d-1}$ be a $\mathbb{Z}_{p}$-basis for the torsion-free part of the subgroup

$$
C=\left\{x \in \mathcal{O}_{K, p}^{\times}: N_{K / \mathbb{Q}}(x)=1\right\} .
$$

The functions $\kappa \mapsto \log \left(\kappa\left(u_{i}\right)\right)$ are analytic functions on $\mathcal{W}^{\text {rig }}$. Moreover, the derivatives of these functions are linearly independent at the origin, and hence anywhere (since they are homomorphisms of rigid-analytic group varieties). Thus they cut out a reduced rigid subvariety of $\mathcal{W}^{\text {rig }}$ of dimension 1 . I claim that every locally algebraic point of $\mathcal{W}(U)$ lies in this subvariety. Indeed, suppose $\kappa$ is such a point, with residue field $E$. Then $\kappa(C)$ must be finite, since the algebraic part of $\kappa$ is trivial on $C$. Therefore $\kappa\left(u_{1}\right), \ldots, \kappa\left(u_{d}\right)$ must be roots of unity in $E^{\times}$; as the subgroup of $C$ generated by the $u_{i}$ is pro- $p$, these must be $p$-power roots of unity. Hence, they are zeros of the $p$-adic logarithm.

On the other hand, the powers of the norm character $\mathcal{O}_{K, p}^{\times} \rightarrow \mathbb{Z}_{p}^{\times}$are in $\mathcal{W}(U)$, and the closure of these (in either the formal or the rigid Zariski topology) is a formal subscheme of $\mathcal{W}$ of dimension 1; so the dimension of the rigid Zariski closure of the locally algebraic weights in $\mathcal{W}(U)$ is exactly 1 .

For the second statement, since $\mathcal{W}(U)=\widehat{Q(U)}$ is affine, it suffices to check that there is no nonzero element of $\mathbb{Z}_{p} \llbracket Q(U) \rrbracket$ whose image under any locally constant character is zero. This is clear since $\mathbb{Z}_{p} \llbracket Q(U) \rrbracket$ is by construction the inverse limit of the $\mathbb{Z}_{p}$-group rings of the finite quotients of $Q(U)$.

We now lift these statements to $\mathcal{E}(U)$. If $\chi$ is a point of $\mathcal{E}(U)$, we say $\chi$ is locally algebraic if its image $\kappa \in \mathcal{W}(U)$ is so (equivalently, if it corresponds to an algebraic Grössencharacter of $K)$; if this is the case, we define $c(\chi)=c(\kappa)$, which is the smallest power of $p$ divisible by the $p$-part of the conductor of the corresponding Grössencharacter.

Proposition 4.3. For any $N<\infty$, the set of locally algebraic points $\chi \in \mathcal{E}(U)$ with $c(\chi)<N$ (or with values in a given coefficient field $E$ ) is contained in a one-dimensional closed formal subscheme of $\mathcal{E}(U)$. The set of all locally algebraic 
points is not contained in any proper closed subscheme of $\mathcal{E}(U)$, but is contained in a one-dimensional closed subvariety of the generic fibre $\mathcal{E}(U)^{\text {rig }}$.

Proof. The map $f: \mathcal{E}(U) \rightarrow \mathcal{W}(U)$ is finite and flat. Hence, the preimage of a closed formal subscheme of $\mathcal{W}(U)$ is a closed formal subscheme of $\mathcal{E}(U)$ of the same dimension. Hence for any $N<\infty$, the preimage of the formal subscheme of Proposition 4.1 is a one-dimensional closed formal scubscheme of $\mathcal{E}(U)$ containing every locally algebraic point $\chi \in \mathcal{E}(U)$ with $c(\chi)<N$. Similarly, the preimage of the subvariety of $\mathcal{W}(U)^{\text {rig }}$ given by Proposition 4.2 is a closed rigid subvariety of $\mathcal{E}(U)^{\text {rig }}$ containing every locally algebraic point.

It remains to show that the set of all locally algebraic points of $\mathcal{E}(U)$ is dense in the formal Zariski topology, or, equivalently, that there is no element of $\mathbb{Z}_{p} \llbracket H(U) \rrbracket$ whose image under every locally algebraic character is zero. As for $\mathcal{W}(U)$ above, this follows from the fact that $\mathbb{Z}_{p} \llbracket H(U) \rrbracket$ is the inverse limit of the $\mathbb{Z}_{p}$-group rings of the finite quotients of $H(U)$; so every nonzero element of the ring $\mathbb{Z}_{p} \llbracket H(U) \rrbracket$ has nonzero image under some locally constant, and hence a fortiori locally algebraic, character.

It follows from the above proposition that for a field $K$ satisfying our assumptions (such as $\mathbb{Q}(\sqrt[3]{2})$ ), a rigid-analytic function on $\mathcal{E}(U)^{\text {rig }}$ is not determined by its values at locally algebraic characters, but that a bounded rigid-analytic function is determined by these values.

Remark 4.1. If $K$ does contain CM subfields, then there is a maximal such subfield $K_{0}$. Let us write $e=1+r_{2}\left(K_{0}\right)+\delta\left(K_{0}\right)$ if such a $K_{0}$ exists, and $e=1$ otherwise. Then we have the following generalization of the above proposition, valid for an arbitrary number field: for any $N$, the (rigid or formal) Zariski closure of the set of locally algebraic points $\chi \in \mathcal{E}(U)$ with $c(\chi)<N$ has dimension at most $e$, as does the rigid Zariski closure of the set of all locally algebraic points. Moreover, this dimension is strictly smaller than $\operatorname{dim} \mathcal{E}(U)$ unless $K$ is either totally real or CM.

\section{Sketch of the $\mathrm{GL}_{2}$ theory}

We now suppose $K$ is an imaginary quadratic field in which $p$ splits, and $\mathfrak{N}$ is an integral ideal of $\mathcal{O}_{K}$ prime to $p$. For integers $a, b \geq 2$, we let $S_{a, b}\left(\Gamma_{1}\left(\mathfrak{N} p^{r}\right)\right)$ denote the space of cuspidal cohomological automorphic forms for $\mathrm{GL}_{2} / K$ of weight $(a, b)$ and level $\Gamma_{1}\left(\mathfrak{N} p^{r}\right)$; equivalently, this is the space $H_{\text {par }}^{1}\left(Y_{1}\left(\mathfrak{N} p^{r}\right), \mathcal{V}_{a, b}\right)$ where $Y_{1}\left(\mathfrak{N} p^{r}\right)$ is the appropriate arithmetic quotient of $\mathrm{GL}_{2}\left(\mathbb{A}_{K}\right)$ and $\mathcal{V}_{a, b}$ is the locally constant sheaf on $Y_{1}\left(\mathfrak{N} p^{r}\right)$ corresponding to the algebraic representation of $\operatorname{Res}_{K / \mathbb{Q}} \mathrm{GL}_{2}$ of highest weight $(a, b)$. This is a finite-dimensional vector space over $K$.

We fix a choice of prime $\mathfrak{p} \mid p$, and hence an embedding $K \hookrightarrow \mathbb{Q}_{p}$. For a locally constant character $\chi$ of $\mathcal{O}_{K, p}^{\times}$of conductor $c$, with values in a finite extension $E$ of $\mathbb{Q}_{p}$, we let $S_{a, b}\left(\Gamma_{1}\left(\mathfrak{N} p^{c}\right), \chi\right)_{\text {ord }}$ denote the subspace of $S_{a, b}\left(\Gamma_{1}\left(\mathfrak{N} p^{c}\right)\right) \otimes_{K} E$ of forms on which the diamond operators act via $\chi$ and which are ordinary at $\mathfrak{p}$ and $\overline{\mathfrak{p}}$.

We say that a locally algebraic weight $\kappa: x \mapsto x^{a} \bar{x}^{b} \varepsilon(x) \in \mathcal{W}$, with $\varepsilon$ of finite order, is arithmetical if $a, b \geq 2$. 
Theorem 5.1 ([8, Theorem 3.2]). There exists a finitely generated $\mathbb{Z}_{p} \llbracket \mathcal{O}_{K, p}^{\times} \rrbracket$-module $\mathbb{H}$ such that for any arithmetical character $\kappa$ as above,

$$
S_{a, b}\left(\Gamma_{1}\left(\mathfrak{N} p^{c}, \varepsilon\right)_{\text {ord }} \cong \mathbb{H} \otimes_{\mathbb{Z}_{p} \llbracket \mathcal{O}_{K, p}^{\times} \rrbracket, \kappa} E .\right.
$$

The sheaf $\mathbb{H}$ on $\mathcal{W}$ corresponds to the pushforward of $\mathcal{O}(\mathcal{E}(U))$ to $\mathcal{W}$ in the $\mathrm{GL}_{1}$ theory. Hida has given a characterization of its geometry analogous to proposition 3.1 above:

Theorem 5.2 ([8, Theorem 6.2]). The support of $\mathbb{H}$ is an equidimensional subscheme of $\mathcal{W}$ of dimension 1.

We also have an obstruction to the existence of locally algebraic points arising from archimedean considerations, analogous to Theorem 4.1:

Theorem 5.3 ([7, Section 3.6.1]). The space $S_{a, b}\left(\Gamma_{1}\left(\mathfrak{N} p^{c}\right)\right)$ is zero unless $a=b$.

Hence, any arithmetical weight lying in Supp $\mathbb{H}$ is locally parallel, and thus contained in a translate by some locally constant character of the one-dimensional subscheme $\mathcal{W}_{0}$ parametrizing parallel weights.

Theorem 5.4 ([2, Lemma 8.8]). There exist triples $(K, \mathfrak{N}, \mathfrak{p})$ such that Supp $\mathbb{H}$ has nonempty intersection with, but does not contain, the component of $\mathcal{W}_{0}$ containing the character $x \mapsto\left(\mathrm{N}_{K / \mathbb{Q}} x\right)^{2}$.

Corollary 5.1. If $(K, \mathfrak{N}, \mathfrak{p})$ is such a triple, then Supp $\mathbb{H}$ has irreducible components $X$ of dimension 1 such that for any $N<\infty$, the set of arithmetical weights $\kappa \in X$ with $c(\kappa)<N$ is finite; in particular, there are finitely many arithmetical weights in $X(E)$ for any given field $E$. Furthermore, the set of all arithmetical weights is not dense in the rigid Zariski topology of Supp $\mathbb{H}$.

Proof. By the preceding lemma, there exists a component $X$ of Supp $\mathbb{H}$ which intersects, but does not equal, the component of $\mathcal{W}_{0}$ containing $x \mapsto\left(\mathrm{N}_{K / \mathbb{Q}} x\right)^{2}$. Hence, it has finite intersection with $\mathcal{W}_{0}$ or any translate of $\mathcal{W}_{0}$ by a locally constant character (since $\operatorname{Spf} \mathcal{W}_{0}$ is quasicompact), which establishes the first claim. For the second claim, we note that the set of all locally parallel weights in $\mathcal{W}$ is the points of a onedimensional closed rigid-analytic subvariety, as in Proposition 4.2, each component of which has finite intersection with $X$; so its intersection with $X$ is not dense in the rigid-analytic Zariski topology of $X$.

However, since the set of locally parallel weights is not formally Zariski-closed in $\mathcal{W}$, one cannot rule out the possibility that the set of all arithmetical locally algebraic weights in $X$ is infinite (and hence dense in $X$ for the formal Zariski topology).

Remark 5.1. It is asserted in [2, Theorem 8.9] that there are components $X$ which contain only finitely many arithmetical weights, but the proof given therein relies on the assertion that the intersection of $X$ with the set of locally parallel weights is formally Zariski closed in $X$ (and hence must be either finite or all of $X$ ); this is false as the set of all locally parallel weights is not formally Zariski closed in $\mathcal{W}$, and the rigid space $\mathcal{W}^{\text {rig }}$ is not quasicompact. Similarly, the arguments of Theorem 7.1 of op.cit. do not show that the Galois-theoretic deformation space constructed therein has finitely many specializations with parallel Hodge-Tate weights, but rather the 
weaker statement that it has finitely many parallel weight specializations which are crystalline at $\mathfrak{p}$ and $\overline{\mathfrak{p}}$ (or become so over any fixed extension of $\mathbb{Q}_{p}$ ).

Remark 5.2. The essential difference between the $\mathrm{GL}_{1}$ and $\mathrm{GL}_{2}$ cases is that in the latter we lack an explicit description of the subscheme Supp $\mathbb{H}$. Thus, in the former case we can show that every component of $\mathcal{E}(U)$ contains infinitely many points corresponding to classical automorphic forms, while in the latter case we cannot rule out the existence of irreducible components of Supp $\mathbb{H}$ containing only finitely many such points - we merely assert that the existence of such components has not been proven.

\section{Acknowledgments}

The author would like to thank Kevin Buzzard for much useful advice, and specifically for alerting him to the results of [11], Frank Calegari for helpful discussions regarding [2], and Sug Woo Shin for his comments on an earlier draft.

\section{References}

[1] K. Buzzard, On p-adic families of automorphic forms, in Modular curves and abelian varieties (Bellaterra, 2002), Vol. 224, Progr. Math., 23-44, Birkhäuser, Basel, 2004.

[2] F. Calegari and B. Mazur, Nearly ordinary Galois deformations over arbitrary number fields, J. Inst. Math. Jussieu 8(1) (2009), 99-177.

[3] R. F. Coleman, Classical and overconvergent modular forms, Invent. Math. 124(1-3) (1996), $215-241$.

[4] R. F. Coleman and B. Mazur, The eigencurve, in Galois representations in arithmetic algebraic geometry (Durham, 1996), Vol. 254, London Math. Soc. Lecture Notes, 1-113, Cambridge University Press, Cambridge, 1998.

[5] A. J. de Jong, Crystalline Dieudonné module theory via formal and rigid geometry, Pub. Math. IHÉS 82 (1995), 5-96.

[6] M. Emerton, On the interpolation of systems of eigenvalues attached to automorphic Hecke eigenforms, Invent. Math. 164(1) (2006), 1-84.

[7] G. Harder, Eisenstein cohomology of arithmetic groups. The case $\mathrm{GL}_{2}$, Invent. Math. 89(1) (1987), 37-118.

[8] H. Hida, p-adic ordinary Hecke algebras for GL(2), Ann. Inst. Fourier (Grenoble) 44(5) (1994), 1289-1322.

[9] D. Loeffler, Overconvergent algebraic automorphic forms, Proc. London Math. Soc. 102(2) (2011), 193-228.

[10] L. Ribes and P. Zalesskii, Profinite groups, Vol. 40, Ergebnisse der Mathematik und ihrer Grenzgebiete. 3. Folge [Results in Mathematics and Related Areas. 3rd Series], Springer-Verlag, Berlin, 2nd edition, 2010.

[11] A. Weil, On a certain type of characters of the idèle-class group of an algebraic number-field, in Proceedings of the International Symposium on Algebraic Number Theory, Tokyo \& Nikko, 1955, 1-7, Science Council of Japan, Tokyo, 1956.

Warwick Mathematics Institute, Zeeman Building, University of Warwick, Coventry CV4 7AL, UK

E-mail address: d.a.loeffler@warwick.ac.uk 